\title{
THE CHOW GROUP MODULO $l$ FOR THE TRIPLE PRODUCT OF A GENERAL ELLIPTIC CURVE*
}

\author{
CHAD SCHOEN ${ }^{\dagger}$
}

0. Introduction. The purpose of this note is to prove

(0.1) TheOREM. Let $\mathbb{Q} \subset k$ be an algebraically closed extension of positive transcendence degree. Let $\mathbf{E}$ be an elliptic curve over $k$ whose $J$-invariant is transcendental over $\mathbb{Q}$. If $l \in\{5,7,11,13,17\}$, then $C H^{2}\left(\mathbf{E}^{3}\right) / l$ is infinite.

The proof is based on a fundamental result of Bloch and Esnault [Bl-Es] which provides a criterion for a nullhomologous cycle to give a non-zero element in the second Chow group modulo $l$. In order to apply the criterion, one must have information concerning the image of the cycle under the $l$-adic Abel-Jacobi map. We will work with the Ceresa cycle [Ce] constructed from a particular genus 3 curve whose Jacobian is isogenous to the triple product of a general elliptic curve. In this case computations of $l$-adic Abel-Jacobi maps were done in [Bu-Sch-Top] using techniques adapted from a paper of Bloch, [Bl]. One may conclude that the class of the Ceresa cycle is not divisible by $l$ in an appropriate Chow group. To show that the Chow group modulo $l$ is indeed infinite, we study the orbit of the Ceresa cycle under the action of modular correspondences as in Nori's paper, [No].

Examples of varieties, $W$, over algebraically closed fields of characteristic 0 and primes $l$ for which $C H^{2}(W) / l$ is infinite were given in [Sch]. The results of that paper apply only to varieties which have complex multiplication cycles or to closely related varieties. Since one may speculate that the Chow group modulo $l$ is infinite for at least a moderately large class of varieties, it seems desirable to have examples which don't depend on the presence of complex multiplication cycles. This paper provides one such example.

Notations. $Z^{r}(W)=$ the group of cycles of codimension $r$ on a separated scheme, $W$, of finite type over a field.

$C H^{r}(W)=$ the group of cycles of codimension $r$ modulo cycles which are rationally equivalent to zero $[\mathrm{Fu}, \S 1]$.

$A[m]:=\operatorname{Ker}: A \stackrel{\cdot m}{\longrightarrow} A$, where $A$ is an abelian group and $\cdot m$ is multiplication by an integer, $m$.

$$
A\left[l^{\infty}\right]:=\cup_{n \in \mathbb{N}} A\left[l^{n}\right] .
$$

1. Overview of the proof. Let $F$ denote the field of rational functions in one variable, $\overline{\mathbb{Q}}(a)$, and let $\bar{F}$ denote its algebraic closure. Define an elliptic curve, $E / F$, by

$$
f_{E}(x, y, z):=y^{2} z-\left[\left(a^{2}-4\right) x^{3}+\left(2 a^{2}-4 a\right) x^{2} z+\left(a^{2}-4\right) x z^{2}\right]=0 .
$$

Theorem (0.1) will follow easily from

(1.2) THEOREM. If $l \in\{5,7,11,13,17\}$, then $C H^{2}\left(E_{\bar{F}}^{3}\right) / l$ is not finite.

Proof. To begin, we use the genus 3 curve,

$$
C: x^{4}+y^{4}+z^{4}+a\left(x^{2} y^{2}+y^{2} z^{2}+z^{2} x^{2}\right)=0,
$$

* Received January 8, 2000; accepted for publication April 5, 2000.

$\dagger$ Department of Mathematics, Duke University, Box 90320, Durham, NC 27708-0320 USA (schoen@math.duke.edu). Partial support by NSF and NSA gratefully acknowledged. 
and the map [Bu-Sch-Top, 4.2],

$$
\pi: C \rightarrow E \text {, }
$$

corresponding to the field extension, $F(E) \rightarrow F(C)$,

$$
x / z \mapsto\left(2(x / z)^{2}+(y / z)^{2}+a\right)^{2}, \quad y / z \mapsto(y / z)\left(2(x / z)^{2}+(y / z)^{2}+a\right),
$$

to construct a cycle on $E^{3}$. The element, $\sigma \in \operatorname{Aut}\left(\mathbb{P}^{2}\right)$, given by

$$
x \circ \sigma=y, \quad y \circ \sigma=z, \quad z \circ \sigma=x,
$$

stabilizes $C$ and gives rise to an embedding,

$$
\varrho: C \rightarrow E^{3}, \quad \varrho=\left(\pi, \pi \circ \sigma, \pi \circ \sigma^{2}\right) .
$$

Define a cycle,

$$
\Xi:=\varrho(C)-(-1)_{*} \varrho(C) \in Z^{2}\left(E^{3}\right) .
$$

It is homologous to zero because $-1 \in \operatorname{Aut}\left(E^{3}\right)$ operates trivially on $H^{4}\left(E_{\bar{F}}^{3}, \mathbb{Z}_{l}(2)\right)$.

The proof of (1.2) now proceeds in three steps:

1. We recall the theorem of Bloch and Esnault [Bl-Es] which provides a criterion for a nullhomologous cycle to give a non-zero class in the Chow group modulo $l$.

2 . We show that the image of a specialization of $\Xi$ under the $l$-adic Abel-Jacobi map is not divisible by $l$. This allows us to use the theorem of Bloch and Esnault to conclude that the class of $\Xi$ in $C H^{2}\left(E_{\bar{F}}^{3}\right) / l$ is not zero.

3. We apply Nori's argument [No] using modular correspondences to create a subgroup, $\left.G \subset \operatorname{Aut}\left(C H^{2}\left(E_{\bar{F}}^{3}\right) / l\right)\right)$, such that the orbit of the class of $\Xi$ under $G$ is not finite.

2. The theorem of Bloch and Esnault. Let $A$ be a smooth projective variety of dimension $d$ defined over $\mathbb{Q}$. Define

$$
C H_{\text {hom }}^{r}(A):=\operatorname{Ker}\left[C H^{r}(A) \rightarrow \prod_{l \text { prime }} H^{2 r}\left(A_{\overline{\mathbb{Q}}}, \mathbb{Z}_{l}(r)\right)\right] .
$$

Set $G_{\mathbb{Q}}=\operatorname{Gal}(\overline{\mathbb{Q}} / \mathbb{Q})$. There is an $l$-adic Abel-Jacobi map [Bu-Sch-Top, $\left.\S 1\right]$,

$$
\mathbf{a}_{l}^{r}: C H_{h o m}^{r}(A) \rightarrow J_{l}^{r}(A, \mathbb{Q}):=H^{1}\left(G_{\mathbb{Q}}, H^{2 r-1}\left(A_{\overline{\mathbb{Q}}}, \mathbb{Z}_{l}(r)\right)\right) .
$$

Write $\overline{\mathbf{a}}_{l}^{r}$ for the composition of $\mathbf{a}_{l}^{r}$ with the tautological map, $J_{l}^{r}(A, \mathbb{Q}) \rightarrow J_{l}^{r}(A, \mathbb{Q}) / l$. Recall the definition of the first level in the coniveau filtration:

$$
N^{1} H^{j}\left(A_{\overline{\mathbb{Q}}}, \mathbb{Z} / l(2)\right):=\operatorname{Ker}\left[H^{j}\left(A_{\overline{\mathbb{Q}}}, \mathbb{Z} / l(2)\right) \rightarrow H^{j}(\overline{\mathbb{Q}}(A), \mathbb{Z} / l(2))\right] .
$$

(2.1) Theorem. Let $A$ be an Abelian variety defined over $\mathbb{Q}$ of dimension d. Let $l$ be a prime number.

(i) The cycle class map of Bloch [B12] gives an isomorphism $\lambda_{l}^{2}: C H^{2}\left(A_{\overline{\mathbb{Q}}}\right)[l] \rightarrow$ $N^{1} H^{3}\left(A_{\overline{\mathbb{Q}}}, \mathbb{Z} / l(2)\right)$.

(ii) If $A$ has good ordinary reduction at $l$ and $d \geq 3$, then $N^{1} H^{3}(A, \mathbb{Z} / l(2)) \neq$ $H^{3}(A, \mathbb{Z} / l(2))$.

Proof. (i) This is a consequence of [Bl2] and [Me-Su,§18] (cf. [Ra, 3.6(2)]). Details may be found in [Sch, 6.1].

(ii) This is [Bl-Es, 1.2] plus [Bl-Ka, 7.4].

Recall that a correspondence, $P \in C H^{d}(A \times A) \otimes \mathbb{Z}_{l}$, acts on Chow groups and cohomology by,

$$
P_{*}(\mathfrak{z}):=p r_{2 *}\left(P \cdot p r_{1}^{*}(\mathfrak{z})\right) \text {. }
$$


This action is compatible with the cycle class map, the coniveau filtration, and the $l$-adic Abel-Jacobi map [Bu-Sch-Top, 1.9(6)].

(2.2) Theorem. Let $A$ be an Abelian variety defined over $\mathbb{Q}$ of dimension $d$ which has good ordinary reduction at a prime, l. Suppose $P_{*} N^{1} H^{3}\left(A_{\overline{\mathbb{Q}}}, \mathbb{Z} / l(2)\right)=0$. If $z \in C H_{\text {hom }}^{2}(A)$ is such that $\overline{\mathbf{a}}_{l}^{2}\left(P_{*} z\right) \neq 0$, then the class of $z$ in $C H_{\text {hom }}^{2}\left(A_{\overline{\mathbb{Q}}}\right) / l$ is not zero.

Proof. This is easily deduced from [Bl-Es, §4]. For details see [Sch, 6.2].

(2.3) Corollary. Let $A$ be an Abelian variety defined over $\mathbb{Q}$ of dimension $d \geq 3$ which has good ordinary reduction at a prime, $l$. Suppose that $P \in C H^{d}(A \times A) \otimes \mathbb{Z}_{l}$ is an idempotent in the ring of correspondences and

(i) $P_{*} H^{3}\left(A_{\overline{\mathbb{Q}}}, \mathbb{Z} / l(2)\right)$ is an irreducible $G_{\mathbb{Q}}$-module,

(ii) $(I d-P)_{*} H^{3}\left(A_{\overline{\mathbb{Q}}}, \mathbb{Z} / l(2)\right)$ is contained in the image of $\lambda_{l}^{2}: C H^{2}\left(A_{\overline{\mathbb{Q}}}\right)[l] \rightarrow$ $H^{3}\left(A_{\overline{\mathbb{Q}}}, \mathbb{Z} / l(2)\right)$,

(iii) there is an element, $z \in C H_{\text {hom }}^{2}(A)$, such that $\overline{\mathbf{a}}_{l}^{2}\left(P_{*} z\right) \neq 0$, then the class of $z$ in $C H_{\text {hom }}^{2}\left(A_{\overline{\mathbb{Q}}}\right) / l$ is not zero.

Proof. By (ii) and (2.1)(i) $(I d-P)_{*} H^{3}\left(A_{\overline{\mathbb{Q}}}, \mathbb{Z} / l(2)\right)=(I d-P)_{*} N^{1} H^{3}\left(A_{\overline{\mathbb{Q}}}, \mathbb{Z} / l(2)\right)$. By $(2.1)$ (ii) $P_{*} N^{1} H^{3}\left(A_{\overline{\mathbb{Q}}}, \mathbb{Z} / l(2)\right) \neq P_{*} H^{3}\left(A_{\overline{\mathbb{Q}}}, \mathbb{Z} / l(2)\right)$. Now (i) and the compatibility of the coniveau filtration with the action of the Galois group implies that $P_{*} N^{1} H^{3}\left(A_{\overline{\mathbb{Q}}}, \mathbb{Z} / l(2)\right)=0$. The corollary follows from $(2.2)$.

3. The nonvanishing of $\Xi$ in $C H_{h o m}^{2}\left(E_{\bar{F}}^{3}\right) / l$. This step in the proof of (1.2) relies heavily on the paper, [Bu-Sch-Top], which is an extension of a fundamental paper of Bloch [Bl].

For fixed $a \in \mathbb{Q}-\{-1, \pm 2\}$ the equation (1.1) defines an elliptic curve, $E_{a}$ over the rational numbers. Similarly (1.3) defines a genus 3 curve over $\mathbb{Q}, C_{a}$, which admits a map, $\pi_{a}: C_{a} \rightarrow E_{a}$, given by (1.4). Formula (1.5) defines a cycle $\Xi_{a} \in Z_{h o m}^{2}\left(E_{a}^{3}\right)$. In [Bu-Sch-Top, 2.3] a correspondence $P \in Z^{3}\left(E_{a}^{3} \times E_{a}^{3}\right)$ is defined. We make this into an idempotent by multiplying by $\frac{1}{3}$. By a mild abuse of notation this idempotent will henceforth be denoted $P$.

(3.1) Proposition. Suppose that $(a, l) \in\left\{(5,5),(1,7),\left(\frac{2}{7}, 11\right),\left(\frac{5}{2}, 13\right),(47,17)\right\}$.

(i) $P_{*} H^{3}\left(E_{a \overline{\mathbb{Q}}}^{3}, \mathbb{Z} / l(2)\right)$ is an irreducible $G_{\mathbb{Q}}$-module.

(ii) $(I d-P)_{*} H^{3}\left(E_{a \bar{Q}}^{3}, \mathbb{Z} / l(2)\right)$ is contained in the image of $\lambda_{l}^{2}: C H^{2}\left(E_{a \overline{\mathbb{Q}}}^{3}\right)[l] \rightarrow$ $H^{3}\left(E_{a \overline{\mathbb{Q}}}^{3}, \mathbb{Z} / l(2)\right)$.

(iii) $\overline{\mathbf{a}}_{l}^{2}\left(P_{*} \Xi_{a}\right) \neq 0$.

(iv) $E_{a}^{3}$ has good ordinary reduction at $l$.

(v) The class of $\Xi_{a}$ in $C H_{h o m}^{2}\left(E_{a \overline{\mathbb{Q}}}^{3}\right) / l$ is non-zero.

Proof. (i) Using the Künneth formula and the definition of $P$ one checks that

$$
P_{*} H^{3}\left(E_{a \overline{\mathbb{Q}}}^{3}, \mathbb{Z} / l(2)\right) \simeq \operatorname{Sym}^{3} H^{1}\left(E_{a \overline{\mathbb{Q}}}, \mathbb{Z} / l\right)(2) .
$$

The action of $G_{\mathbb{Q}}$ on $H^{1}\left(E_{a \overline{\mathbb{Q}}}, \mathbb{Z} / l\right)$ gives rise to a representation, $\kappa: G_{\mathbb{Q}} \rightarrow G L(2, \mathbb{Z} / l)$. The third symmetric power of the tautological representation of $S L(2, \mathbb{Z} / l)$ is irreducible for primes $l \geq 5$. Thus one need only show that $\kappa\left(G_{\mathbb{Q}}\right)$ contains $S L(2, \mathbb{Z} / l)$. Let $\bar{\kappa}\left(G_{\mathbb{Q}}\right)$ denote the image of $\kappa\left(G_{\mathbb{Q}}\right)$ in $P G L(2, \mathbb{Z} / l)$. According to [La, p.183 and p.185] if $\kappa\left(G_{\mathbb{Q}}\right)$ does not contain $S L(2, \mathbb{Z} / l)$, then either $\kappa\left(G_{\mathbb{Q}}\right)$ is contained in a Borel subgroup of $G L(2, \mathbb{Z} / l)$ or the order of $\kappa\left(G_{\mathbb{Q}}\right)$ is prime to $l$ and one of the following holds:

- $\bar{\kappa}\left(G_{\mathbb{Q}}\right)$ is cyclic and $\kappa\left(G_{\mathbb{Q}}\right)$ is contained in a Cartan subgroup.

- $\bar{\kappa}\left(G_{\mathbb{Q}}\right)$ is dihedral and $\kappa\left(G_{\mathbb{Q}}\right)$ is contained in the normalizer of a Cartan sub- 
group.

- $\bar{\kappa}\left(G_{\mathbb{Q}}\right)$ is isomorphic to $A_{4}, S_{4}$, or $S_{5}$.

In the second row of the following table we give for each pair, $(a, l)$, a prime $p$ of good reduction for $E_{a}$ with the property that the geometric Frobenius, $\kappa\left(F r o b_{p}^{-1}\right)$, lies in a split Cartan subgroup and the order of $\kappa\left(F r o b_{p}^{-1}\right), \mathbf{o}_{p}$, is the same as the order of its image in $P G L(2, \mathbb{Z} / l)$. In the third row a prime $p$ is given such that $\kappa\left(F_{r o b}{ }_{p}^{-1}\right)$ lies in a non-split Cartan subgroup of $G L(2, \mathbb{Z} / l)$ and has the indicated order, $\mathbf{o}_{p}$. This information is easily deduced from the characteristic polynomial of Frob $_{p}^{-1}, t^{2}-a_{p} t+p$, where $a_{p}=1+p-\left|E_{a}\left(\mathbb{F}_{p}\right)\right|$. The software, GP-PARI, was used to compute $a_{p}$.

\begin{tabular}{|l|l|l|l|l|l|}
\hline$(a, l)$ & $(5,5)$ & $(1,7)$ & $(2 / 7,11)$ & $(5 / 2,13)$ & $(47,17)$ \\
\hline \hline$\left(p, \mathbf{o}_{p}\right)$ & $(13,4)$ & $(13,3)$ & $(17,10)$ & $(5,12)$ & $(19,8)$ \\
\hline$\left(p, \mathbf{o}_{p}\right)$ & $(43,24)$ & $(5,48)$ & $(5,60)$ & $(19,168)$ & $(11,96)$ \\
\hline
\end{tabular}

One deduces easily from the table that $S L(2, \mathbb{Z} / l) \subset \kappa\left(G_{\mathbb{Q}}\right)$ for each choice of $(a, l)$.

(ii) The symmetric group, $\mathcal{S}_{3}$, acts on $E_{a}^{3}$ by permuting the factors. Using the Künneth formula one may decompose $H^{3}\left(E_{a \overline{\mathbb{Q}}}^{3}, \mathbb{Z} / l(2)\right)$ as the sum of $\operatorname{Sym}^{3} H^{1}\left(E_{a \overline{\mathbb{Q}}}, \mathbb{Z} / l\right)(2)$ and the $\mathbb{Z} / l\left[\mathcal{S}_{3}\right]$-module, $H$, generated by

$$
H^{0}\left(E_{a \overline{\mathbb{Q}}}, \mathbb{Z} / l\right) \otimes H^{2}\left(E_{a \overline{\mathbb{Q}}}, \mu_{l}\right) \otimes H^{1}\left(E_{a \overline{\mathbb{Q}}}, \mu_{l}\right) \quad \text { and } \quad \Delta \cdot H^{1}\left(E_{a \overline{\mathbb{Q}}}, \mu_{l}\right),
$$

where $\Delta$ generates $\Lambda^{2} H^{1}\left(E_{a \overline{\mathbb{Q}}}^{2}, \mathbb{Z} / l\right)(1) \subset H^{2}\left(E_{a \overline{\mathbb{Q}}}^{2}, \mu_{l}\right)$. Now using the isomorphism from the Kummer sequence, $C H^{1}\left(E_{a \overline{\mathbb{Q}}}\right)[l] \simeq H^{1}\left(E_{a \overline{\mathbb{Q}}}, \mu_{l}\right)$, and the fact that $H^{0}\left(E_{a \overline{\mathbb{Q}}}, \mathbb{Z} / l\right) \otimes H^{2}\left(E_{a \overline{\mathrm{Q}}}, \mu_{l}\right) \oplus \Delta \mathbb{Z} / l$ is in the image of the cycle class map, N.S. $\left(E_{a}^{2}\right) \rightarrow$ $H^{2}\left(E_{a \bar{Q}}, \mu_{l}\right)$, one sees that $H$ is in the image of $\lambda_{l}^{2}$. To complete the proof, note that $(I d-P)_{*} H=(I d-P)_{*} H^{3}\left(E_{a \overline{\mathbb{Q}}}^{3}, \mathbb{Z} / l(2)\right)$.

(iii) The proceedure used to calculate $\overline{\mathbf{a}}_{l}^{2}\left(P_{*} \Xi_{a}\right)$ is described in [Bu-Sch-Top, §35]. To summarize briefly, one reduces $E_{a \mathbb{Q}}^{3}$ modulo an appropriate prime $p$ of good reduction. One then computes

$$
p_{3 *}\left(D \cdot P_{*} \Xi_{a \mathbb{F}_{p}}\right)_{l} \in C H^{1}\left(E_{a \mathbb{F}_{p}}\right)\left[l^{\infty}\right]
$$

for $D$ a suitable divisor in $E_{a \mathbb{F}_{p}}^{3}$, where $p_{3}: E_{a \mathbb{F}_{p}}^{3} \rightarrow E_{a \mathbb{F}_{p}}$ is projection on the third factor, the subscript, $l$, means take the $l$-primary component of a torsion element in the Chow group, and $\Xi_{a \mathbb{F}_{p}}$ is the nullhomologous cycle obtained by reducing $\Xi_{a}$ modulo $p$. The association, $P_{*} \Xi_{a} \mapsto p_{3 *}\left(D \cdot P_{*} \Xi_{a \mathbb{F}_{p}}\right)_{l}$, factors through the $l$-adic Abel-Jacobi map [Bu-Sch-Top, 4.8(1)], so if

$$
C H^{1}\left(E_{a \mathbb{F}_{p}}\right)\left[l^{\infty}\right]=C H^{1}\left(E_{a \mathbb{F}_{p}}\right)[l],
$$

then $p_{3 *}\left(D \cdot P_{*} \Xi_{a \mathbb{F}_{p}}\right)_{l} \neq 0$ implies $\overline{\mathbf{a}}_{l}^{2}\left(P_{*} \Xi_{a}\right) \neq 0$. The actual calculation of $p_{3 *}(D$. $\left.P_{*} \Xi_{a \mathbb{F}_{p}}\right)$ is sufficiently involved to require a computer. The results of these calculations for the triples, $(a, l, p) \in\{(5,5,13),(1,7,29),(2 / 7,11,41),(5 / 2,13,43),(47,17,73)\}$ (and for numerous others) are summarized in [Bu-Sch-Top, Table 9.2]. In each of the five cases $p_{3 *}\left(D \cdot P_{*} \Xi_{a F_{p}}\right)_{l} \neq 0$. Also (3.2) holds because in each case the Riemann hypothesis implies that no elliptic curve over the field $\mathbb{F}_{p}$ can have a point of order $l^{2}$. 
(iv) It suffices to show that $E_{a}$ has good ordinary reduction at $l$. Since the discriminant of the Weierstrass model for $E_{a}$ in [Bu-Sch-Top, p. 113] is 256(a+1) $(a+$ $2)^{4}(-a+2)^{6}$ it is easy to check that $E_{a}$ has good reduction at $l$. To show that it has ordinary reduction one need only check that $\left|E_{a}\left(\mathbb{F}_{l}\right)\right| \neq l+1$ [Sil, V.Ex5.10].

(v) This follows from (2.3).

(3.3) Proposition. If $l \in\{5,7,11,13,17\}$, then the class of $\Xi$ in $C H_{h o m}^{2}\left(E_{\bar{F}}^{3}\right) / l$ is non-zero.

Proof. For any finite extension, $F \subset F^{\prime}$, there is a specialization map, $C H^{2}\left(E_{F^{\prime}}^{3}\right) \rightarrow$ $C H^{2}\left(E_{a \overline{\mathbb{Q}}}^{3}\right)$. Passing to the direct limit gives a specialization map, $C H^{2}\left(E_{\bar{F}}^{3}\right) \rightarrow$ $C H^{2}\left(E_{a \bar{Q}}^{3}\right)$. There is also a specialization map on etale cohomology and the two specialization maps are compatible with the cycle class map [Fu, 20.3.5]. Thus specialization gives a map,

$$
C H_{h o m}^{2}\left(E_{\bar{F}}^{3}\right) / l \rightarrow C H_{h o m}^{2}\left(E_{a \bar{Q}}^{3}\right) / l,
$$

which sends $\Xi$ to $\Xi_{a}$. Given $l \in\{5,7,11,13,17\}$, we may chose $a \in \mathbb{Q}$ such that $(3.1)(\mathrm{v})$ implies that the class of $\Xi_{a}$ in $C H_{h o m}^{2}\left(E_{a \overline{\mathbb{Q}}}^{3}\right) / l$ is non-zero. The proposition follows.

4. The action of modular correspondences on $C H^{2}\left(E_{\bar{F}}^{3}\right) / l$. In this final step in the proof of (1.2) we use modular correspondences to create a subgroup, $G \subset$ $\operatorname{Aut}\left(\mathrm{CH}^{2}\left(E_{\bar{F}}^{3}\right) / l\right)$, such that the orbit of the class of $\Xi$ is infinite. The argument is taken from [No] with modifications added to adapt it to the present context.

The first task is to relate the elliptic curve $E$ of (1.1) to the universal elliptic curve over a modular curve. The quadratic twist, $\mathcal{E}$, of $E$ by the field extension,

$$
F \subset F^{\prime}:=F(\sqrt{2-a}),
$$

is the elliptic curve over $F$ defined by $f_{\mathcal{E}}(x, y, z)=0$, where

$$
f_{\mathcal{E}}(x, y, z)=y^{2} z+\left[(a+2) x^{3}+(2 a) x^{2} z+(a+2) x z^{2}\right] .
$$

Note that $f_{\mathcal{E}}$ is obtained from $f_{E}$ in (1.1) by dividing all terms except the first by $(2-a)$.

(4.2) LEMMA. $\mathcal{E}$ is isomorphic to the generic fiber of the universal elliptic curve with a point of order 4 .

Proof. There is a fine moduli space for elliptic curves with a point of order 4 [Ed, 4.3]. We denote it by $\dot{X}_{1}(4)$ and its compactification by $X_{1}(4)$. It is a degree 6 cover of the $J$-line. The point of order $4,(-1: 2: 1) \in \mathcal{E}(F)$, gives rise to a morphism from $\mathcal{E}$ to the universal elliptic curve over $\dot{X}_{1}(4)$. The $J$-invariant of $\mathcal{E}$ is

$$
J=-16 \frac{\left(a^{2}-12 a-12\right)^{3}}{(a+2)^{4}(a+1)} .
$$

As $[F: \overline{\mathbb{Q}}(J)]=6$, it follows that $F \simeq \overline{\mathbb{Q}}\left(X_{1}(4)\right)$ and $\mathcal{E}$ is isomorphic to the generic fiber of the universal family of elliptic curves with a point of order 4 .

Write $\phi: E_{\bar{F}} \rightarrow \mathcal{E}_{\bar{F}}$ for the isomorphism induced by applying Proj to the $\bar{F}$ algebra homomorphism,

$$
\bar{F}[x, y, z] /\left(f_{\mathcal{E}}\right) \rightarrow \bar{F}[x, y, z] /\left(f_{E}\right), \quad x \mapsto x, \quad y \mapsto(2-a)^{-1 / 2} y, \quad z \mapsto z .
$$

Define $[\Xi]:=\phi_{*}^{3}(\Xi) \in Z_{\text {hom }}^{2}\left(\mathcal{E}_{\bar{F}}^{3}\right)$.

We now turn to the construction of modular correspondences on $\mathcal{E}_{\bar{F}}$. 
Let $N \in \mathbb{N}$ be odd, square free and prime to $l$. Let $X_{0}(N)$ denote the compactification of the coarse moduli space for the functor, elliptic curves with a cyclic subgroup of order $N$. Write $X_{0}(4 N)$ for the normalization of the fiber product over the $J$-line, $X_{1}(4) \times_{\mathbb{P}^{1}} X_{0}(N)$. (The notation, $X_{0}(4 N)$, agrees with standard notation because the tautological map, $X_{1}(4) \rightarrow X_{0}(4)$, is an isomorphism.) The curve, $\dot{X}_{0}(4 N)$, obtained by removing the cusps from $X_{0}(4 N)$ represents the functor, elliptic curves with a point of order 4 and a cyclic subgroup of order $N$. Let $\varsigma_{N}: \mathcal{E}_{N} \rightarrow \dot{X}_{0}(4 N)$ denote the universal elliptic curve. It is isomorphic to the pullback of the universal elliptic curve over $\dot{X}_{1}(4)$ by the "forget the level $N$-structure" map, $h_{N}: \dot{X}_{0}(4 N) \rightarrow \dot{X}_{1}(4)$. Let $p$ be a prime dividing $N$. Write $H_{N} \subset \mathcal{E}_{N}$ for the cyclic subgroup of order $N$ and $H_{N}(p) \subset H_{N}$ for the subgroup of order $p$. The elliptic curve, $\mathcal{E}_{N} / H_{N}(p)$, inherits a point of order 4 from $\mathcal{E}_{N}$ and has a cyclic subgroup of order $N$ given by $\left(\mathcal{E}_{N}[p] / H_{N}(p)\right) \times\left(H_{N} / H_{N}(p)\right)$. Thus it may be realized as a pullback of the universal elliptic curve over $\dot{X}_{0}(4 N)$. This gives a commutative diagram in which the square on the right is Cartesian and the map on the upper left is tautological:

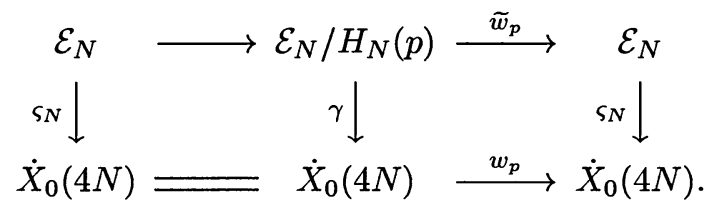

Write $u_{p}: \mathcal{E}_{N} \rightarrow \mathcal{E}_{N}$ for the map in the top row of (4.4). Let $m_{n} \in \operatorname{End}\left(\mathcal{E}_{N}\right)$ denote multiplication by $n$ and let $\epsilon: \mathbb{Z} \rightarrow\{ \pm 1\}$ denote the quadratic Dirichlet character of conductor 4 .

(4.5) LEMMA. $w_{p}^{2}=I d$ and $u_{p} \circ u_{p}=m_{\epsilon(p) p}$.

Proof. Taking the quotient in the right hand square of (4.4) by subgroups of order $p$ gives rise to the left hand square in the Cartesian diagram,

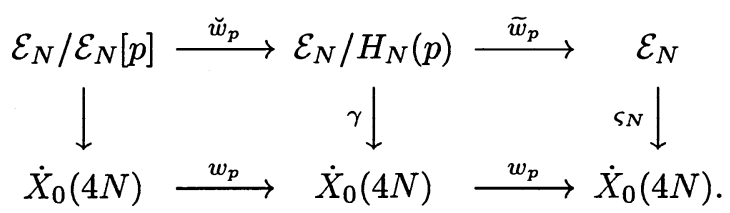

Observe that $\mathcal{E}_{N} / \mathcal{E}_{N}[p]$ has a level $4 N$ structure consisting of the point of order 4 which comes from the tautological point of order 4 on $\mathcal{E}_{N}$ via the canonical quotient map and the cyclic subgroup of order $N$ which is the image of

$$
\left(H_{N} / H_{N}(p)\right) \times\left(\mathcal{E}_{N} / H_{N}(p)\right)[p] \subset \mathcal{E}_{N} / H_{N}(p)
$$

under the map, $\mathcal{E}_{N} / H_{N}(p) \rightarrow \mathcal{E}_{N} / \mathcal{E}_{N}[p]$. One checks immediately that $\widetilde{w}_{p} \circ \breve{w}_{p}$ maps this level $4 N$ structure to the tautological level $4 N$ structure on $\mathcal{E}_{N}$. Since both squares in (4.6) are Cartesian, the map, $w_{p} \circ w_{p}$, is the map to the fine moduli space, $\dot{X}_{0}(4 N)$, induced by the level $4 N$ structure on $\mathcal{E}_{N} / \mathcal{E}_{N}[p]$. Since $\mathcal{E}_{N} / \mathcal{E}_{N}[p]$ has a unique cyclic subgroup of order $N$ and a unique cyclic subgroup of order 4 , one of the two isomorphisms of $\dot{X}_{0}(4 N)$-schemes, $\xi: \mathcal{E}_{N} / \mathcal{E}_{N}[p] \rightarrow \mathcal{E}_{N}$, must preserve the level $4 N$ structure. Thus $w_{p}^{2}=I d$. When we compose the canonical quotient map, $\mathcal{E}_{N} \rightarrow \mathcal{E}_{N} / \mathcal{E}_{N}[p]$, with the isomorphism, $\xi$, preserving the level $4 N$ structure, we get $m_{ \pm p}$ with the sign determined by $p \bmod 4$.

Set $F_{N}:=\overline{\mathbb{Q}}\left(X_{0}(4 N)\right)$. For $N \mid N^{\prime}$ there is a natural inclusion, $F_{N} \subset F_{N^{\prime}}$, corresponding to the map, forget the level $N^{\prime} / N$-structure, $X_{0}\left(4 N^{\prime}\right) \rightarrow X_{0}(4 N)$. Thus 
there is a tower of fields,

$$
F \subset F_{N} \subset F_{N^{\prime}} \subset \ldots,
$$

which we embedd in $\bar{F}$. Set $F_{\infty}:=\cup_{N} F_{N} \subset \bar{F}$, where the union is over square free, odd $N$ prime to $l$. For each odd prime $p$ distinct from $l$ and multiple, $N$, of $p$, there is an involution $w_{p} \in \operatorname{Aut}\left(\operatorname{Spec}\left(F_{N}\right)\right)$. If $N \mid N^{\prime}$, then $w_{p} \in \operatorname{Aut}\left(\operatorname{Spec}\left(F_{N^{\prime}}\right)\right)$ induces $w_{p} \in \operatorname{Aut}\left(\operatorname{Spec}\left(F_{N}\right)\right)$. This gives rise to an involution of $\operatorname{Spec}\left(F_{\infty}\right)$ denoted by $w_{p}$ as well. Fix a lifting, $\bar{w}_{p} \in \operatorname{Aut}(\operatorname{Spec}(\bar{F}))$, of $w_{p}$. For $N$ a multiple of $p$ define a map of schemes,

$$
\bar{u}_{p}: \mathcal{E}_{\bar{F}} \simeq \mathcal{E}_{N} \times_{\dot{X}_{0}(4 N)} \operatorname{Spec}(\bar{F}) \stackrel{u_{p} \times \bar{w}_{p}}{\longrightarrow} \mathcal{E}_{N} \times_{\dot{X}_{0}(4 N)} \operatorname{Spec}(\bar{F}) \simeq \mathcal{E}_{\bar{F}},
$$

which makes sense because both $u_{p}$ and $\bar{w}_{p}$ are compatible with the map, $w_{p}$, on $\dot{X}_{0}(4 N)$. The map is independent of the choice of $N$. Similarly there is a map,

$$
U_{p}:=\bar{u}_{p} \times \bar{u}_{p} \times \bar{u}_{p}: \mathcal{E}_{\bar{F}}^{3} \rightarrow \mathcal{E}_{\bar{F}}^{3},
$$

because $\bar{u}_{p}$ is compatible with $\bar{w}_{p} \in \operatorname{Aut}(\operatorname{Spec}(\bar{F}))$. Now $U_{p}$ induces maps on Chow groups and etale cohomology which are compatible with the cycle class map. Thus it gives rise to an element, $U_{p}^{*} \in \operatorname{End}\left(C H_{\text {hom }}^{2}\left(\mathcal{E}_{\bar{F}}^{3}\right) / l\right)$.

(4.7) Lemma. $U_{p}^{*}$ is invertible.

Proof. Recall the exact sequence,

$$
0 \rightarrow C H_{\text {alg }}^{2}\left(\mathcal{E}_{\bar{F}}^{3}\right) \rightarrow C H_{\text {hom }}^{2}\left(\mathcal{E}_{\bar{F}}^{3}\right) \rightarrow \operatorname{Griff}^{2}\left(\mathcal{E}_{\bar{F}}^{3}\right) \rightarrow 0,
$$

defining the Griffiths group as the quotient of $\mathrm{CH}_{\text {hom }}^{2}$ by the subgroup generated by cycles algebraically equivalent to zero. As $C H_{\text {alg }}^{2}\left(\mathcal{E}_{\bar{F}}^{3}\right)$ is generated by Jacobians of curves via correspondences, it is a divisible group. Thus

$$
C H_{h o m}^{2}\left(\mathcal{E}_{\bar{F}}^{3}\right) / l \simeq \operatorname{Griff}^{2}\left(\mathcal{E}_{\bar{F}}^{3}\right) / l .
$$

The map,

$$
\bar{u}_{p}^{\prime}:=u_{p} \times \bar{w}_{p}^{-1}: \mathcal{E}_{N} \times_{X_{0}(4 N)} \operatorname{Spec}(\bar{F}) \rightarrow \mathcal{E}_{N} \times_{X_{0}(4 N)} \operatorname{Spec}(\bar{F}),
$$

is well defined since both $u_{p}$ and $\bar{w}_{p}^{-1}$ are compatible with $w_{p} \in \operatorname{Aut}\left(\dot{X}_{0}(4 N)\right)$. Furthermore $\bar{u}_{p} \circ \bar{u}_{p}^{\prime}$ may be identified with multiplication by $\epsilon(p) p$ in $\operatorname{End}\left(\mathcal{E}_{\bar{F}}\right)$ by (4.5). Write $M_{n} \in \operatorname{End}\left(\mathcal{E}_{\bar{F}}^{3}\right)$ for multiplication by $n$. Set $U_{p}^{\prime}:=\left(\bar{u}_{p}^{\prime}\right)^{3}$. Then $U_{p} \circ U_{p}^{\prime}=M_{\epsilon(p) p}$. Now $M_{\epsilon(p) p *} \circ M_{\epsilon(p) p}^{*}$ acts by multiplication by $p^{6}=\operatorname{deg}\left(M_{\epsilon(p) p}\right)$ on $C H\left(\mathcal{E}_{\bar{F}}^{3}\right)$ by the projection formula. On the other hand, $M_{\epsilon(p) p}^{*} \circ M_{\epsilon(p) p *}$ takes a cycle to the sum of its translates by the points of order $p$ on $\mathcal{E}_{\bar{F}}^{3}$. It thus acts on $\operatorname{Griff}^{2}\left(\mathcal{E}_{\bar{F}}^{3}\right)$ by multiplication by $p^{6}$. Since $p \neq l, M_{\epsilon(p) p}$ acts invertibly on $C H_{\text {hom }}^{2}\left(\mathcal{E}_{\bar{F}}^{3}\right) / l$. Hence $U_{p}$ does as well.

(4.9) Remark. In fact both $M_{\epsilon(p) p}^{*}$ and $M_{\epsilon(p) p *}$ act by multiplication by $\epsilon(p) p^{3}$ on $\operatorname{Griff}^{2}\left(\mathcal{E}_{\bar{F}}^{3}\right) \otimes \mathbb{Z}\left[\frac{1}{2}\right]$ [Bl-Sri, $\S 4$ Proposition].

Let $\mathcal{P}=\{p$ prime $: p \equiv 1 \bmod 4 l\}$.

(4.10) THEOREM. The set $\mathfrak{U}:=\left\{U_{p}^{*}[\Xi] \in C H_{\text {hom }}^{2}\left(\mathcal{E}_{\bar{F}}^{3}\right) / l: p \in \mathcal{P}\right\}$ is infinite.

Proof. The set $\mathcal{P}$ is infinite by Dirichlet's theorem on primes in an arithmetic progression. We will show that the classes $U_{p}^{*}[\Xi] \in C H_{h o m}^{2}\left(\mathcal{E}_{\bar{F}}^{3}\right) / l$ for $p \in \mathcal{P}$ are linearly independent over $\mathbb{Z} / l$ by studying the action of $G a l\left(\bar{F} / F_{\infty}\right)$ on $C H_{h o m}^{2}\left(\mathcal{E}_{\bar{F}}^{3}\right) / l$.

Recall $F^{\prime}=F(\sqrt{2-a})$. Define $F_{N}^{\prime}:=F^{\prime} F_{N}$ and $F_{\infty}^{\prime}:=F^{\prime} F_{\infty} \subset \bar{F}$. Write $\chi$ for the composition,

$$
\operatorname{Gal}\left(\bar{F} / F_{\infty}\right) \rightarrow \operatorname{Gal}\left(F_{\infty}^{\prime} / F_{\infty}\right) \simeq\{ \pm 1\}
$$


Let $\chi_{p}$ denote the character which results when $\bar{w}_{p}\left(F_{\infty}^{\prime}\right)$ is substituted for $F_{\infty}^{\prime}$ in the above expression. In other words, $\chi_{p}(g)=\chi\left(\bar{w}_{p} g \bar{w}_{p}^{-1}\right)$.

Now (4.10) is an immediate consequence of

(4.11) Proposition.

(i) Each $g \in \operatorname{Gal}\left(\bar{F} / F_{\infty}\right)$ acts on $[\Xi] \in Z^{2}\left(\mathcal{E}_{\bar{F}}^{3}\right)$ by $g[\Xi]=\chi(g)[\Xi]$.

(ii) For $p \in \mathcal{P}$ the action of each $g \in \operatorname{Gal}\left(\bar{F} / F_{\infty}\right)$ on $C H_{\text {hom }}^{2}\left(\mathcal{E}_{\bar{F}}^{3}\right) / l$ satisfies

$$
g U_{p}^{*}[\Xi]=\chi_{p}(g) U_{p}^{*}[\Xi] .
$$

(iii) For distinct primes $p \in \mathcal{P}$ the characters, $\chi_{p}: \operatorname{Gal}\left(\bar{F} / F_{\infty}\right) \rightarrow\{ \pm 1\}$, are distinct.

Proof. (i) The Galois group, $\operatorname{Gal}\left(\bar{F} / F_{\infty}\right)$, acts on $E_{\bar{F}}$ and $\mathcal{E}_{\bar{F}}$ by acting on the coefficients in (4.3). For $g \in \operatorname{Gal}\left(\bar{F} / F_{\infty}\right)$ we have,

$$
g \circ \phi=\phi \circ \chi(g) I d_{E} \circ g
$$

where $\chi(g) I d_{E} \in \operatorname{Aut}\left(E_{\bar{F}}\right)$ denotes inversion in the group law if $\chi(g)=-1$ and the identity if $\chi(g)=1$. Since inversion in the group law on $E_{\bar{F}}^{3}$ acts by -1 on $\Xi \in Z^{2}\left(E_{\bar{F}}^{3}\right)$ and $\operatorname{Gal}(\bar{F} / F)$ fixes this element, (i) follows.

(ii) From

$\bar{u}_{p} g \bar{u}_{p}^{\prime}=\left(u_{p} \times \bar{w}_{p}\right) \circ(I d \times g) \circ\left(u_{p} \times \bar{w}_{p}^{-1}\right)=\left(u_{p} \circ u_{p}\right) \times \bar{w}_{p} g \bar{w}_{p}^{-1}=m_{\epsilon(p) p} \times \bar{w}_{p} g \bar{w}_{p}^{-1}$

we deduce

$$
U_{p} g U_{p}^{\prime}=M_{\epsilon(p) p} \circ\left(I d \times \bar{w}_{p} g \bar{w}_{p}^{-1}\right) .
$$

By (4.8) and (4.9) $M_{\epsilon(p) p}$ acts as the identity on $C H_{\text {hom }}^{2}\left(\mathcal{E}_{\bar{F}}^{3}\right) / l$ for $p \in \mathcal{P}$. Taking $g=$ $I d$ in the previous equation gives that $U_{p}^{\prime}$ acts as the inverse of $U_{p}$ on $C H_{\text {hom }}^{2}\left(\mathcal{E}_{\bar{F}}^{3}\right) / l$. Thus

$$
\left(U_{p}^{*}\right)^{-1} g U_{p}^{*}=\left(U_{p} g U_{p}^{\prime}\right)^{*}=\left(\bar{w}_{p} g \bar{w}_{p}^{-1}\right)^{*} \in \operatorname{End}\left(C H_{h o m}^{2}\left(\mathcal{E}_{\bar{F}}^{3}\right) / l\right)^{*} .
$$

Now (ii) follows from (i) by applying (4.12) to $[\Xi]$.

(iii) Write $\dot{X}^{\prime} \rightarrow \dot{X}_{1}(4)$ for the proper map of irreducible curves corresponding to the field extension, $F \subset F^{\prime}=F(\sqrt{2-a})$. The branch locus, $B \subset \dot{X}_{1}(4)$, corresponds to the parameter value, $a=2$. Write $Q$ for the elliptic curve over $\mathbb{Q}$ obtained by setting $a=2$ in (4.1). The $J$-invariant of $Q$ is $2^{11} 3^{-1}$. As it is not an integer, $Q$ does not have complex multiplication [Sil2,II.6].

Let $p_{1}$ and $p_{2}$ be distinct odd primes. Then $\bar{w}_{p_{1}}\left(F_{\infty}^{\prime}\right)$ and $\bar{w}_{p_{2}}\left(F_{\infty}^{\prime}\right)$ are quadratic extensions of $F_{\infty}$. They coincide exactly when $\bar{w}_{p_{1}}(\sqrt{2-a}) \in \bar{w}_{p_{2}}\left(F_{\infty}^{\prime}\right)$. Since $\bar{w}_{p_{2}}\left(F_{\infty}^{\prime}\right)=\lim _{N} \bar{w}_{p_{2}}\left(F_{N}^{\prime}\right)$, this means $\bar{w}_{p_{1}}(\sqrt{2-a}) \in \bar{w}_{p_{2}}\left(F_{N}^{\prime}\right)$ for some $N$ divisible by $p_{1} p_{2}$. Equivalently $\bar{w}_{p_{1}}\left(F_{N}^{\prime}\right)=\bar{w}_{p_{2}}\left(F_{N}^{\prime}\right)$. The quadratic extension, $F_{N} \subset$ $\bar{w}_{p_{i}}\left(F_{N}^{\prime}\right)=F_{N}\left(\bar{w}_{p_{i}}(\sqrt{2-a})\right)$, corresponds to a degree two map of algebraic curves, $\tau_{N, p_{i}}: \dot{X}_{N, p_{i}}^{\prime} \rightarrow \dot{X}_{0}(4 N)$, which is branched at $w_{p_{i}}\left(h_{N}^{-1}(B)\right)$. To show $\bar{w}_{p_{1}}\left(F_{N}^{\prime}\right) \neq$ $\bar{w}_{p_{2}}\left(F_{N}^{\prime}\right)$ it suffices to show $w_{p_{1}}\left(h_{N}^{-1}(B)\right) \neq w_{p_{2}}\left(h_{N}^{-1}(B)\right)$. In fact we claim that $w_{p_{1}}\left(h_{N}^{-1}(B)\right) \cap w_{p_{2}}\left(h_{N}^{-1}(B)\right)=\emptyset$. The fiber of $\varsigma_{N}: \mathcal{E}_{N} \rightarrow \dot{X}_{0}(4 N)$ over a point in $w_{p_{i}}\left(h_{N}^{-1}(B)\right)$ is isomorphic to $Q / H_{i}$, where $H_{i} \subset Q$ is a subgroup of order $p_{i}$. If subgroups $H_{i}$ of order $p_{i}$ were to exist such that $Q / H_{1} \simeq Q / H_{2}$, then there would be an endomorphism,

$$
\nu: Q \rightarrow Q / H_{1} \simeq Q / H_{2} \stackrel{\tau}{\rightarrow} Q
$$


where the kernel of $\tau$ is $Q\left[p_{2}\right] / H_{2}$ and the kernel of the $\nu$ has order $p_{1} p_{2}$. This contradicts the observation that $Q$ does not have complex multiplication and proves the claim. This completes the proof of (4.11) and (4.10).

Proof of Theorem (1.2). Since $H^{4}\left(\mathcal{E}_{\bar{F}}^{3}, \mathbb{Z}_{l}(2)\right)$ is torsion free, $\operatorname{Tor}\left(\frac{\mathbb{Z}}{l}, \frac{C H^{2}\left(\mathcal{E}_{\bar{F}}^{3}\right)}{C H_{h o m}^{2}\left(\mathcal{E}_{\bar{F}}^{3}\right)}\right)$ $=0$. Thus $C H_{\text {hom }}^{2}\left(\mathcal{E}_{\bar{F}}^{3}\right) / l$ infinite implies that $C H^{2}\left(\mathcal{E}_{\bar{F}}^{3}\right) / l$ is infinite.

Proof of Theorem (0.1). Let $k$ be an algebraically closed field of positive transcendence degree over $\mathbb{Q}$. Let $\mathbf{E}_{k}$ be an elliptic curve over $k$ whose $J$-invariant, $J \in k$, is transcendental over $\mathbb{Q}$. Write $\mathbf{E}$ for an elliptic curve over $\overline{\mathbb{Q}(J)}$ which becomes isomorphic to $\mathbf{E}_{k}$ after extension of scalars. There is an isomorphism, $\overline{\mathbb{Q}(J)} \simeq \bar{F}$, which lifts to an isomorphism, $\mathbf{E} \simeq \mathcal{E}_{\bar{F}}$. Now (0.1) follows from (1.2) and the fact that the Chow group modulo $l$ is unchanged under base extension by algebraically closed fields [Le].

(4.13) Remark. The methods of this section do not apply directly to elliptic curves over $\overline{\mathbb{Q}}$ because modular correspondences do not give self maps of elliptic curves over $\overline{\mathbb{Q}}$. There is one instance known to the author in which $C H^{2}\left(\mathbf{E}^{3}\right) / l$ has been shown to be infinite for an elliptic curve, $\mathbf{E}$, defined over $\overline{\mathbb{Q}}$. In this case the $J$-invariant is 0 [Sch, §10].

(4.14) Remark. An application of Theorem (0.1) is given in [Sch2].

\section{REFERENCES}

[Bl] S. BLOCH, Algebraic cycles and values of L-functions, J. f. reine u. angew. Math., 350 (1984), pp. 94-108.

[B12] S. BLOCH, Torsion algebraic cycles and a theorem of Roitman, Compositio Math., 39 (1979), pp. 107-127.

[Bl-Es] S. BLOCH AND H. ESNAULT, The coniveau filtration and non-divisibility for algebraic cycles, Math. Ann., 304 (1996), pp. 303-314.

[Bl-Ka] S. Bloch AND K. Kato, p-adic etale cohomology, Publ. Math. IHES, 63 (1986), pp. 107-152.

[Bl-Sri] S. Bloch And V. SRINIVAs, Remarks on Correspondences and Algebraic Cycles, Am. J. Math., 105 (1983), pp. 1235-1253.

[Bu-Sch-Top] J. Buhler, C. Schoen, AND J. Top, Cycles, L-functions and productes of elliptic curves, J. reine angew. Math., 492 (1997), pp. 93-133.

[Ce] G. CeresA, $C$ is not algebraically equivalent to $C^{-}$in its Jacobian, Ann. Math., 117 (1983), pp. 285-291.

[Ed] B. Edixhoven, The modular curves $X_{0}(N)$, notes for a series of lectures at the 1997 ICTP Summer school (available from the author's web site, http://www.maths. univ-rennes1.fr/ edix/cours/trieste.html).

[Fu] W. Fulton, Intersection Theory, Springer-Verlag, New York, 1984.

[La] S. LANG, Introduction to Modular Forms, Grundlehren der Math. Wissen. 222, Springer-Verlag, New York, 1976.

[Le] F. Lecomte, Rigidité des groupes de Chow, Duke Math. J., 53 (1986), pp. 405-426.

[Me-Su] A. Mercurev AND A. Suslin, K-cohomology of Severi-Brauer varieties and the norm residue homomorphism, Math. USSR Izv., 21 (1983), pp. 307-340 (English translation).

[No] M. NoRI, Cycles on the generic abelian threefold, Proc. Indian Acad. Sci. (Math. Sci.) 99 (1989), pp. 191-196.

[Ra] W. RASKIND, Algebraic K-theory, Etale cohomology and torsion algebraic cycles, Contemp. Math., 83 (1989), pp. 311-341.

[Sch] C. SCHOen, Complex varieties for which the Chow group mod $n$ is not finite, to appear in J. Alg. Geom..

[Sch2] C. Schoen, On certain exterior product maps of Chow groups, Mathematical Research 
Letters, 7 (2000), pp. 177-194.

[Sil] J. Silverman, The Arithmetic of Elliptic Curves, Springer-Verlag, New York, 1986.

[Sil2] J. Silverman, Advanced Topics in the Arithmetic of Elliptic Curves, Springer-Verlag, New York, 1994. 